ÁREA TEMÁTICA: LICENCIAMENTO AMBIENTAL

\title{
LICENCIAMENTO AMBIENTAL APLICADO ÀS TELECOMUNICAÇÕES: UM ESTUDO DE CASO COM ESTAÇÕES RÁDIOS-BASE DE SERVIÇO MÓVEL NO MUNICÍPIO DE BELÉM
}

Rodrigo Otávio Silva da Costa- eng.rodrigocosta1@ gmail.com

Universidade Federal Rural da Amazônia

Sarah Dias Azevedo - saahazevedo@gmail.com

Universidade Federal Rural da Amazônia

Wanderley Augusto da Silva Pinto - wanderleysilva1503@gmail.com

Universidade Federal Rural da Amazônia

Paulo Rick Soares Rodrigues - - rickpaulo83@gmail.com

Universidade Federal Rural da Amazônia

Leandro Marques Torres - leandrotorres15@yahoo.com.br

Universidade Federal Rural da Amazônia

Deborá Prissila Reis Sandim - deborasandim@gmail.com

Universidade Federal Rural da Amazônia

Fábio Haruki Hatano - fhatano@ gmail.com

Universidade Federal Rural da Amazônia

Glauber Tadaiesky Marques - gtadaiesky@ hotmail.com

Universidade Federal Rural da Amazônia

Arthur Magno - arturmagno15@outlook.com

Secretaria Municipal de Meio Ambiente de Belém

Carnot Luiz Braun Guimarães - carnot@anatel.gov.br

Agência Nacional das Telecomunicações

Adailton Jesus Pereira Lima - adailtonp@ anatel.gov.br

Agência Nacional das Telecomunicações

José Felipe Souza de Almeida - wirelinux@ gmail.com

Universidade Federal Rural da Amazônia 


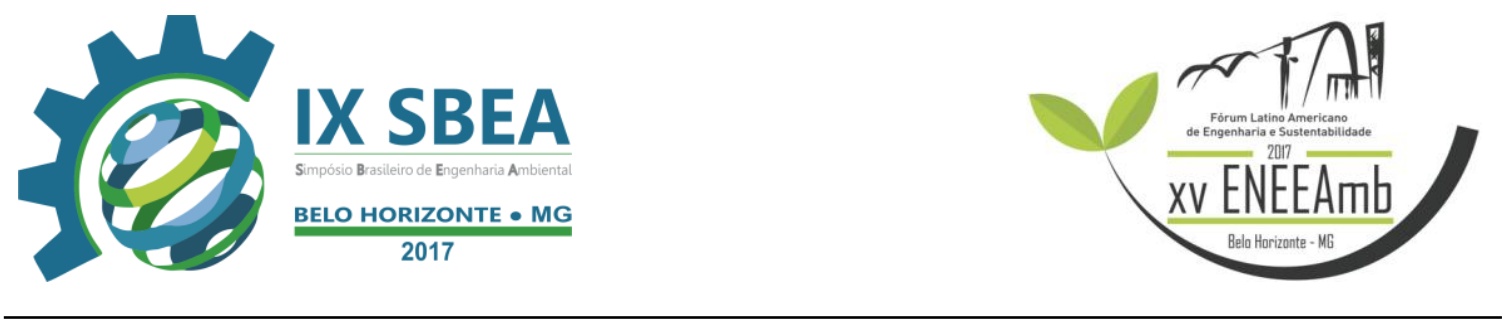

\section{RESUMO}

$\mathrm{O}$ artigo tem foco principal a análise qualitativa e quantitativa de Estações Radio Bases licenciadas ambientalmente em Belém. A metodologia aplicada para a obtenção dos dados deu-se a partir de visitações a SEMMAS (Secretaria Municipal de Meio Ambiente e Sustentabilidade), ANATEL (Agência Nacional das Telecomunicações) a instalações de antenas pela cidade. Com isto, serão dissertados os limites estabelecidos pela Anatel; como as radiações emitidas podem interferir no cotidiano e a análise desses dados. Durante a discussão, são relatadas as faixas máximas de campo elétrico, magnético e densidade de potência que uma pessoa propensa a eles pode receber normalmente. Além de ressaltar o "porquê" das antenas serem instaladas a mais de 30 metros de escolas, creches, asilos, ou qualquer local de aglomeração excedente. Será discorrido os efeitos térmicos e "não térmicos" dessas exposições e qual será a influência na saúde das pessoas. Portanto, nas nossas considerações, concluímos que na capital ocorre pouca fiscalização por parte da SEMMAS, visto que apenas $10 \%$ do total de ERBs possuem licenciamento ambiental.

Palavras-chave: Radiações não-ionizantes. Telefonia Móvel. Torres de Telecomunicações

\section{INTRODUÇÃO/OBJETIVO}

$\mathrm{O}$ presente estudo tem como tema o Licenciamento Ambiental aplicado às Telecomunicações: um estudo de caso com estações rádios-base no município de Belém. Nesse contexto, é de suma importância que todas as ERBs, estrutura presente no APÊNDICE I, sejam licenciadas ambientalmente, apesar da normativa municipal, que trate desse tipo de licenciamento seja relativamente nova (implementada em 2008), a qual tem como objetivo disciplinar o processo de Licenciamento Ambiental de Estações Rádio Base (ERBs) e de equipamentos de Telefonia sem fio, no município de Belém (SEMMA, 2008). O processo utilizado para a realização do presente estudo será o método hipotético/dedutivo, de natureza quantitativa e qualitativa, tendo por base pesquisa de campo e revisão bibliográfica (MARCONI; LAKATOS, 2010). Para tanto, estabelecemos como hipóteses a serem analisadas: 


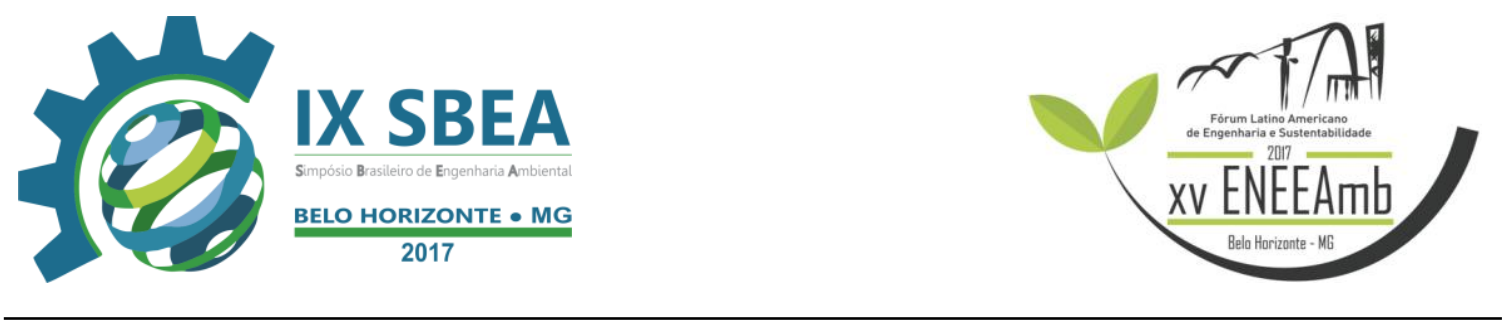

a) Nem todas as ERBs instaladas em Belém são licenciadas ambientalmente;

b) A atividade das ERB's afeta a vida cotidiana das pessoas que moram no entorno delas.

De acordo com BRASIL (2016) existem cerca de 625 ERBs instaladas em Belém. Por isso, diante dessa realidade, o foco principal será analisar quantiqualitativamente as estações rádio-base de telefonia móvel licenciadas ambientalmente, visto que atualmente é vivenciado uma fase de crescimento intensivo da utilização de telefonia celular (PESSOA, 2009).

Devido ao aumento no quantitativo de ERBs, ao longo destes anos, por conta da velocidade com que foi implantado e aperfeiçoado o serviço móvel pessoal no Brasil, como em outros países, isso afetou consideravelmente os hábitos de comunicação da sociedade, a qual se acostumou e incorporou uma revolucionária tecnologia de transmissão de dados e informações ao dia-a-dia sem uma devida discussão e realização de um estudo científico concreto sobre os potenciais impactos ambientais que poderiam ser gerados a partir de disso (PESSOA, 2009).

Nessa conjuntura, temos como nosso objetivo geral analisar as Estações Rádio Base instaladas em Belém-PA, quantitativamente e qualitativamente no que diz respeito aos aspectos da legislação vigente e do Licenciamento ambiental, caracterizando-as no enquadramento destas. Mais especificamente, queremos quantificar o percentual de Estações Rádio Base licenciadas e não licenciadas ambientalmente, analisar a instrução normativa $n^{\circ}$ 002/2008, que trata do licenciamento de ERBs no município de Belém, diagnosticar os impactos ambientais gerados pelas ERBs e discutir os efeitos da radiação não ionizantes provocados pelas ERBs de telefonia celular.

\section{METODOLOGIA}

Buscaram-se fontes especializadas na temática Licenciamento Ambiental, avaliaçao de impactos ambientais e legislações municipais sobre licenciamento ambiental de ERBs e frequências utilizadas por ERBs por meio da consulta de livros, periódicos, teses, dissertações e da legislação existente sobre telefonia móvel, emissões eletromagnéticas e ERB's. 


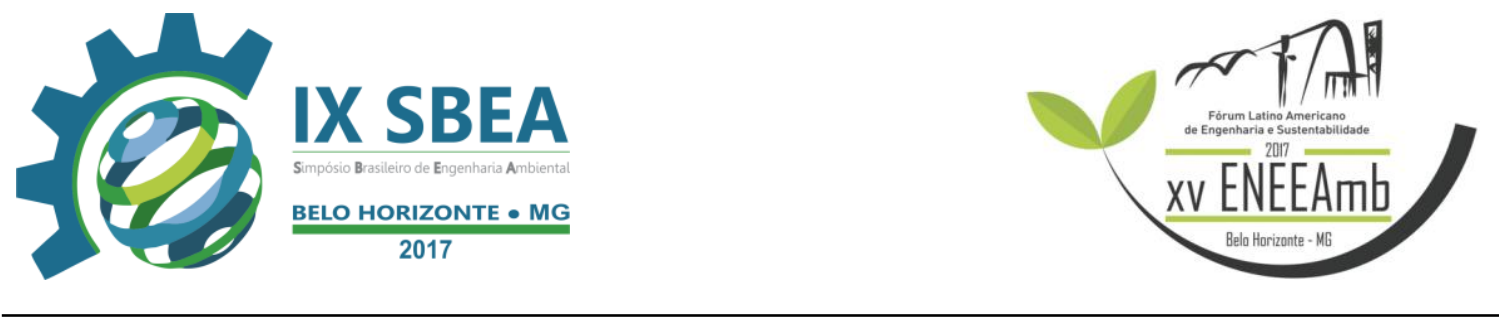

Além disso, foram realizadas coletas de dados na ANATEL e SEMMAS no período de março a maio de 2016, com o objetivo de saber o quantitativo de ERBs instaladas em Belém, bem como saber o quantitativos de ERBs licenciadas ambientalmente. A partir da semana do 11 de abril foram realizadas visitas a ERBs previamente selecionadas, respeitando, sobretudo, a escala de trabalho do Sr. Adailton, gerente de fiscalização da ANATEL, para fazer a coleta das informações de campo elétrico, magnético e densidade de potência.

Ademais, objetivando a aquisição dos dados, os quais mensurem os níveis de radiação emitida pelas antenas no perímetro dos locais escolhidos para análise, foram feitas medições com a utilização da sonda INSTRONIC, instrumento utilizado para medição do campo eletromagnético. A medição do campo eletromagnético se deu na direção de seu maior ganho, ou seja, direção de máxima radiação (SANTARÉM, 2012).

O equipamento fornece valores referentes à intensidade do campo elétrico observado no perímetro dos locais a serem analisados, conforme a figura 01. A partir desse valor é possível se calcular a intensidade do campo magnético e a densidade de potência irradiada (BRASIL, 2002).

$\mathrm{S}=\mathrm{E}^{2} / 377$ e $\mathrm{H}=\mathrm{E} / 377$

Onde:

S é a densidade de potência, em $\mathrm{W} / \mathrm{m}^{2}$;

E é a intensidade do campo elétrico, em $\mathrm{V} / \mathrm{m}$;

H é a intensidade do campo magnético, em $\mathrm{A} / \mathrm{m}$;

377 é o valor da impedância de espaço livre, em Ohms
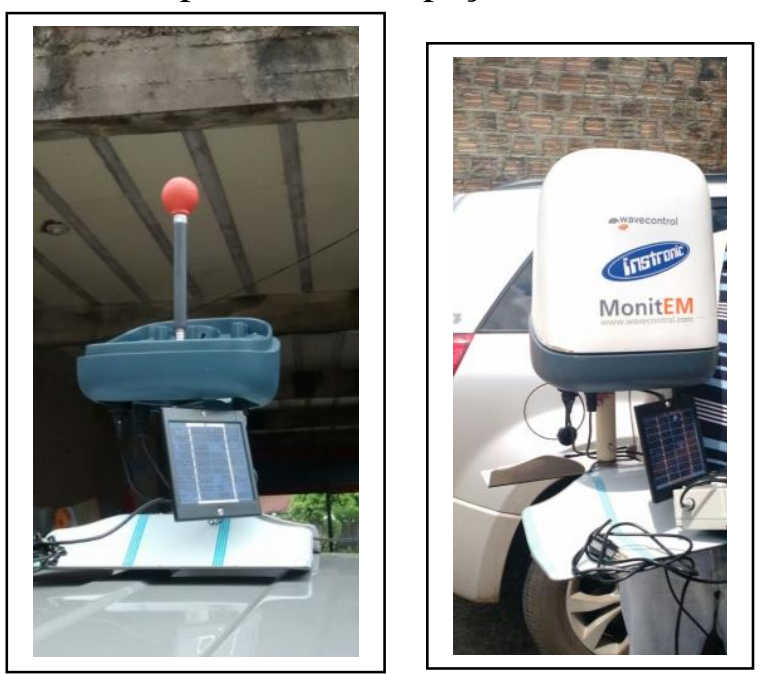


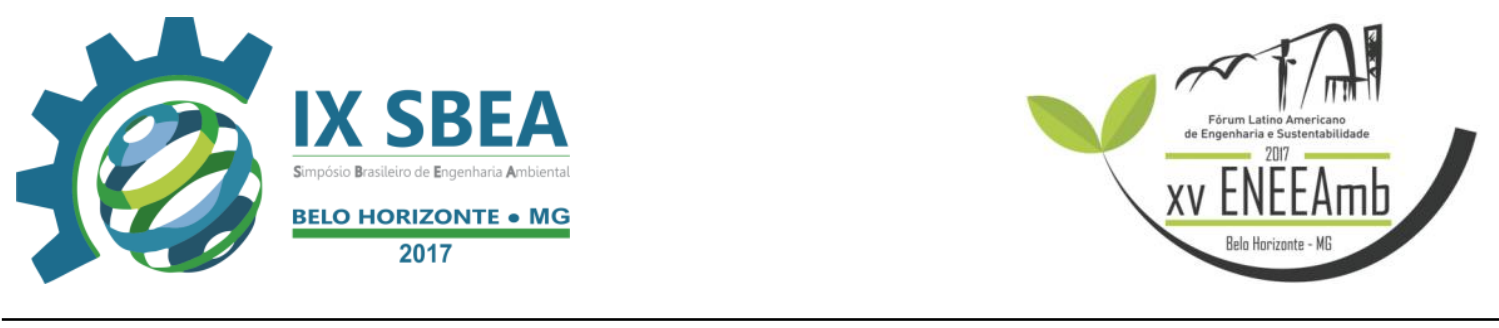

Figura 1: Representação do analisador espectro utilizado na pesquisa

Fonte: Os autores, 2016

Após todas as demais etapas cumpridas, serão realizadas a tabulação dos resultados da pesquisa cientifica e confecção do artigo final do projeto.

\section{RESULTADOS E DISCUSSÃO}

Os dados mostram que existem, na cidade de Belém, cerca de 625 ERBs. Verificou-se, neste estudo, que as principais operadoras atuantes na cidade são: Vivo, Claro, OI, Tim e Nextel (ABT, 2015). A distribuição percentual das ERBs por operadora está mostrada na Figura 0. Observa-se que a operadora com maior percentual de torres na cidade é a TIM (32,2\%), e a Nextel foi a empresa com menor percentual de torres, perfazendo apenas 3,68\% destas. As empresas, CLARO, OI VIVO apresentaram percentuais semelhantes $(18,4 \%, 23,2 \%, 22,8 \%$, respectivamente) (ABT, 2015).

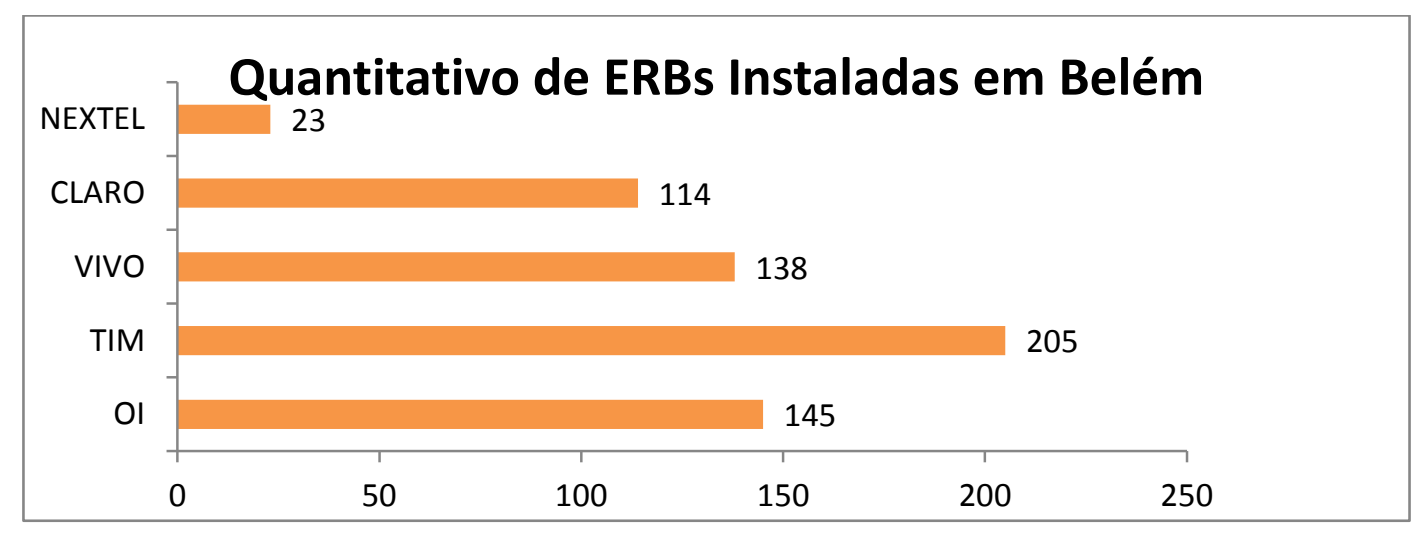

Figura 05: Quantitativo de ERBs instaladas em Belém.

Fonte: Anatel, 2016

A Figura 3 mostra o quantitativo de antenas em percentagem que possui licenciamento ambiental junto a SEMMAS. Analisando a figura 3, notamos que a cidade de Belém licenciou pouquíssimas ERB's desde que a legislação foi criada, tendo apenas $10 \%$ do seu total licenciado, sendo que nos anos de 2008, 2009, 2010, 2013 e 2014 não houveram licenciamentos na cidade. Tais dados trazem certas preocupações quanto a eficiência da prefeitura em fiscalizar a ERB's, tendo em vista a necessidade de análise dessas antenas para garantir qualidade de vida aos moradores adjacentes. Esses resultados mostram que precisam ser realizadas fiscalizações mais ostensivas em torres 


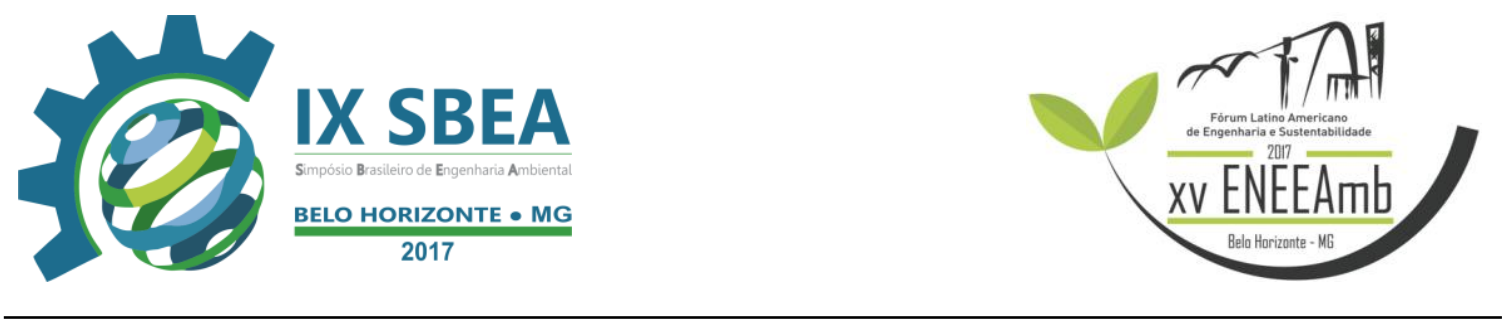

não licenciadas ambientalmente, bem como contratação de mais profissionais capacitados para estarem realizando as fiscalizações, uma vez que nos processos de licenciamento ambiental, uma vez que é feito uma consulta à população local num raio de $100 \mathrm{~m}$ da torre a ser licenciada.

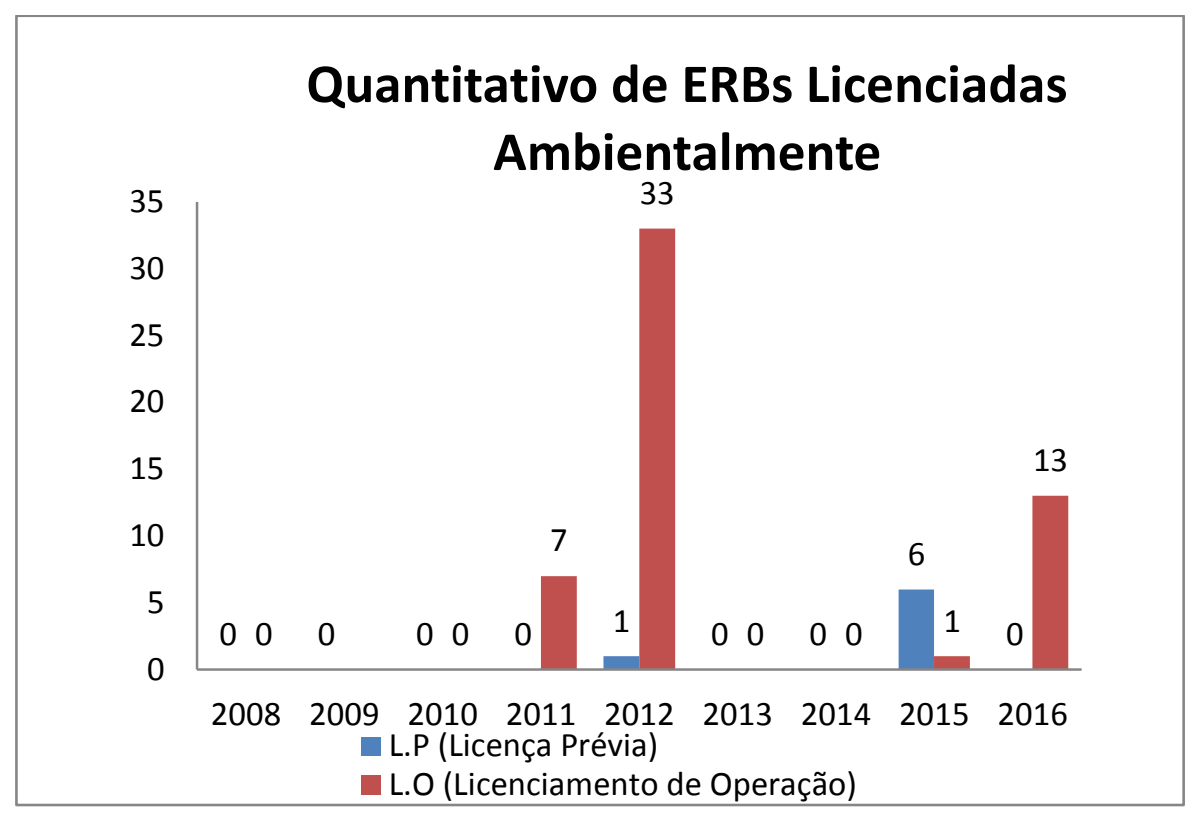

Figura 03: Quantitativo de ERBs licenciadas ambientalmente

Fonte: Semmas, 2016

No âmbito da análise da legislação vigente a instrução normativa ${ }^{\circ}$ 002/2008, é a única legislação que fixa parâmetros para instalação e operação de ERBs. Nela é contemplado pontos imprescindíveis tais como: a localização e distância mínima entre torres, além da exigência de análise prévia de estudos ambientais, laudos técnicos emitidos pela operadora, pareceres conclusivos e licenças da SEMMAS.

Ainda convém lembrar que a distância mínima estabelecida pela SEMMAS para instalação de ERBs de serviço móvel pessoal próximas a unidades escolares creches, unidades hospitalares e asilos é de 30 metros (SEMMAS, 2008).Tal justificativa é plausível, pelo fato de que nestes locais os indivíduos permanecem por períodos extensos, dessa forma, potencialmente mais expostos à radiação não-ionizante. 


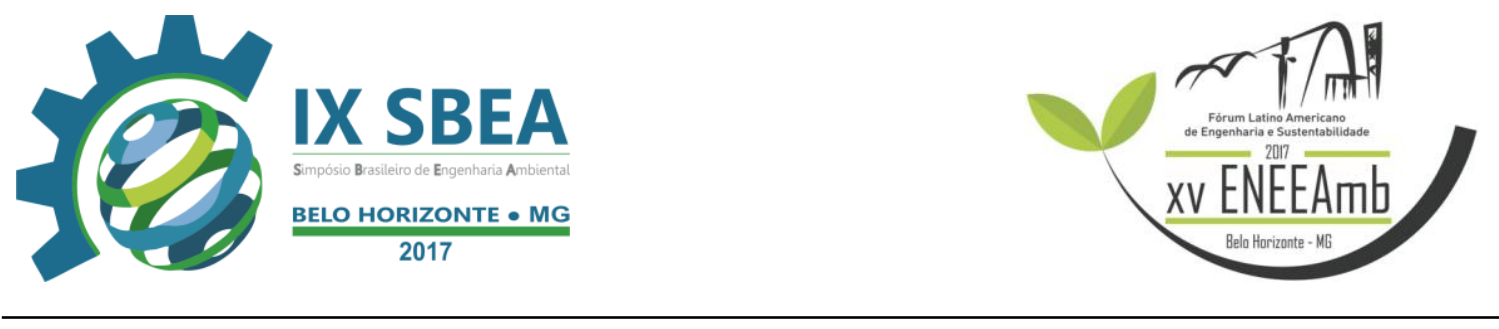

Deve se levar em consideração que tais locais apresentam um grande aglomerado de pessoas, encaixando-se também em outro parâmetro relativo ao licenciamento, aumento assim a preocupação quanto à exposição humana a altos níveis de radiação neste local, desta forma a instalação de ERB's em tais localizações deve ser vistas com cautela.

No entanto, os resultados apresentados no apêndice II e III foram obtidos após registros de radiação com períodos de 6 (seis) minutos, atendendo aos critérios normativos de BRASIL (2002) para este tipo de levantamento de dados, conforme a tabela 01. Todos os dados pesquisados são referentes a escolas e hospitais, que ficam nas imediações das ERB's.

Tabela 01: Limites máximos de radiações não ionizantes aceitáveis pela ANATEL.

Fonte: BRASIL, 2002.

\begin{tabular}{|c|c|}
\hline Descrição & $\begin{array}{c}\text { Limite Máximo para População } \\
\text { Geral }\end{array}$ \\
\hline Limite Exposição ao Campo Elétrico (V/m) & 41,25 \\
\hline Limite de Exposição a Campo Magnético (A / m) & 1,11 \\
\hline Limite de Densidade de Potência (W / m2). & 4,50 \\
\hline
\end{tabular}

Nota-se que os resultados obtidos, em todos os locais pesquisados, para intensidade do campo elétrico, magnético e densidade de potência irradiada, estão bem abaixo dos limites estabelecidos pela ANATEL. Estas estações rádios-base devem ser licenciadas e precisam ser avaliadas pelo órgão direcionado e responsável da adaptação urbanística. As normas implantadas pelas redes de telefonia celular necessitam disciplinar a organização dos equipamentos de molde, sem conter poluição visual (MARCHESAN, 2004). 


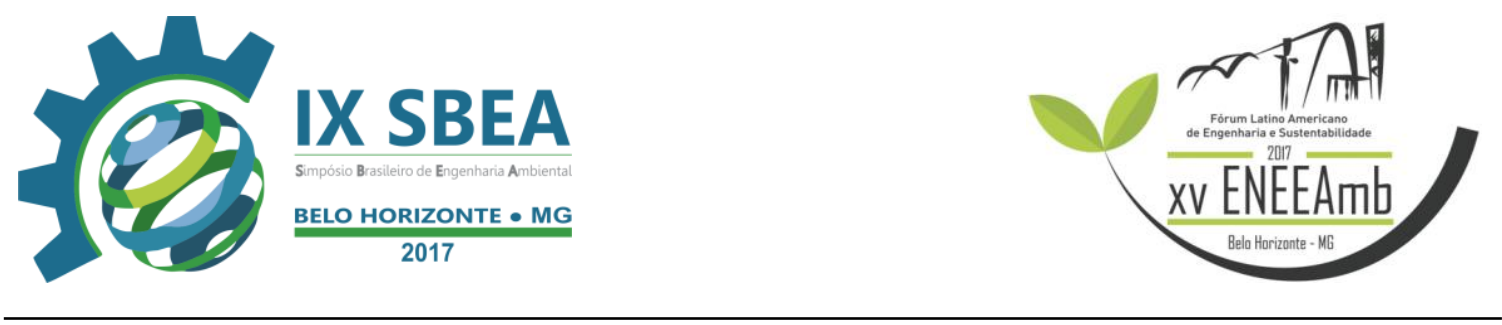

Em relação à distância mínima entre ERBs, a norma estabelece 200 metros. Entretanto, conforme dados obtidos na SEMMAS (2016), esta é a principal irregularidade encontrada nas vistorias realizadas para o licenciamento ambiental. Fato demonstrado, conforme o mapa do apêndice IV obtido pela ABT (2016), que mostra a distribuição das ERBs em Belém. Tal fato deve ser analisado com cautela, uma vez que a instalação das antenas teve início no final da década de 90, de acordo com a ANATEL e a instrução normativa foi aprovada somente no 2 de janeiro de 2008.

Outro aspecto a ser analisado é a zona de efeito combinatório, que consiste na somatória das frequiências utilizadas pelas operadoras instaladas em um determinado local. Porém de acordo com informações obtidas na ANATEL e, também, medições de campos eletromagnéticos realizadas em escolas e hospitais, que ficam localizadas nestas zonas, os resultados ficam abaixo dos valores estipulados pela Resolução 303/2002 da ANATEL.

Além disso, o referido Estudo de Impacto de Vizinhança (EIV) e o seu respectivo Relatório de Impacto de Vizinhança (RIV) são imprescindíveis para o licenciamento ambiental, de acordo a instrução normativa, tal estudo deve ser realizado em um raio de 100 metros a partir do eixo da torre. Esses elementos são de fundamental importância, pois eles qualificam as áreas de atuação (direta ou indireta), análise sócioambiental (caracterização social, econômica e cultural) e diagnósticos dos impactos de vizinhança (SEMMAS, 2008).

Outro ponto a se considerar é com relação à radiação eletromagnética, a qual é a energia emitida por uma determinada fonte, a uma determinada frequência, onde os campos eletromagnéticos são parte do espectro da radiação. Para que os efeitos possam ser estudados é necessário, antes de tudo, saber o que são as radiações eletromagnéticas não ionizantes. As ondas de rádio utilizadas ERB's, assim como a luz visível e as micro-ondas, são radiações não ionizantes. O efeito que a radiação não ionizante provoca é o aumento de temperatura que são os efeitos térmicos e não térmicos (FERNÁNDEZ; SALLES, 2004). 


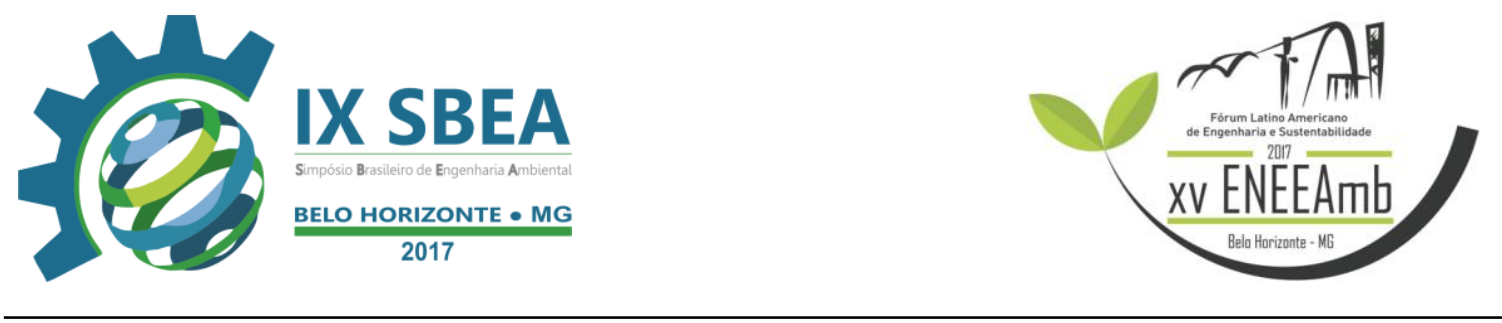

Existe, ainda, o temor de que as consequências dos efeitos não térmicos da exposição aos campos eletromagnéticos da telefonia celular afetem a saúde, intervindo no sistema imunológico ou causando mudanças na atividade cerebral e, também, provocando o câncer. Com relação aos efeitos não térmicos, a ciência não encontrou um conjunto de evidências suficientemente testadas e comprovadas, revisadas e validadas para justificar alterações nos limites de exposição recomendados internacionalmente pelo Comitê Internacional de Proteção da Radiação Não Ionizante (ICNIRP) (BRASIL, 2002).

\section{CONCLUSÕES/RECOMENDAÇÕES}

O presente artigo buscou contribuir para o aumento do conhecimento sobre como são tratadas as estações rádios bases em Belém, e a partir dele mostrou-se que o município possui uma ineficaz fiscalização nas estações; pois mesmo tendo uma legislação vigente, a capital possui apenas de $10 \%$ de ERBs licenciadas ambientalmente. Contudo, mostra que é necessário aprimorar os métodos que realizam as fiscalizações. A SEMMAS, principal órgão deste segmento, deve assegurar e intensificar um maior percentual quantitativo e qualitativo das estações para que estejam de acordo com os dizeres da ANATEL. Outra consideração a ser feita é a partir dos estudos apresentados houve poucas divergências ou variações entre os dados das emissões de raios não-ionizantes pelas ERB's; o que conclui que não há qualquer indício de prejuízo à saúde humana em relação ao princípio de precaução.

\section{REFERÊNCIAS BIBLIOGRÁFICAS}

ABT. Associação Brasileira das Telecomunicações. Disponível em: < http://www.telebrasil.org.br/panorama-do-setor/mapa-de-erbs-antenas> Acessado em: 4 de abril de 2016.

BRASIL. Agência Nacional das Telecomunicações. Disponível em: http://sistemas.anatel.gov.br/easp/Novo/Consulta/Tela.asp?OP=E\&SISQSmodulo=1658 7 Acessado em: 02 de abril de 2016. 
BRASIL. Agência Nacional Das Telecomunicações. Resolução 303: Regulamenta o Limite de exposição a campos elétricos, magnéticos e eletromagnéticos na faixa de radiofrequência entre 9 kHz e 300 GHz. Brasil. De 02 de julho de 2002.

FERNANDÉZ, C.R; SALLES, A.A. O Impacto Das Radiações Não Ionizantes Da Telefonia Móvel E O Princípio Da Precaução. Caderno jurídico, v.6, n.2, p. 22, 2004. MARCHESAN, A.M.M. As estações de rádio-base de telefonia celular no contexto de uma sociedade de riscos. Caderno jurídico, v.6, n.2, p. 148, 2004.

MARCONI, M. de A.; LAKATOS, E. M. Fundamentos de metodologia científica. 7. Ed. São Paulo: Atlas, 2010.

PESSOA, M. B. A Utilização da Telefonia Móvel no Ambiente Urbano: Um Estudo Sócio-Jurídico e Ambiental sobre as Estações Rádio-Base em João Pessoa / Pb. Dissertação (Mestrado em Desenvolvimento e Meio Ambiente.) - Universidade Federal da Paraíba, João Pessoa, 2009.

SANTARÉM. E.W.P. Poluição Ambiental dos Campos Eletromagnéticos de Estações Radio, Televisão e Estações de Radio Base na Telefonia Celular de Manaus. Dissertação (Mestrado em Sustentabilidade na Amazônia.) - Universidade Federal do Amazonas, Manaus, 2012.

SEMMA. Secretaria Municipal de Meio Ambiente de Belém. Termo De Referência Para O Licenciamento Ambiental De Estações Rádiobase - Erb’s E De Equipamentos De Telefonia Sem Fio. Disponível em: < http://www.belem.pa.gov.br/semma/site/wp-content/uploads/2015/09/TR-ESTACOESRADIO-BASE.pdf> Acessado em 02 de abril de 2016. 
APÊNDICE I: Representação da estrutura de uma Estação Rádio Base

ANTENA

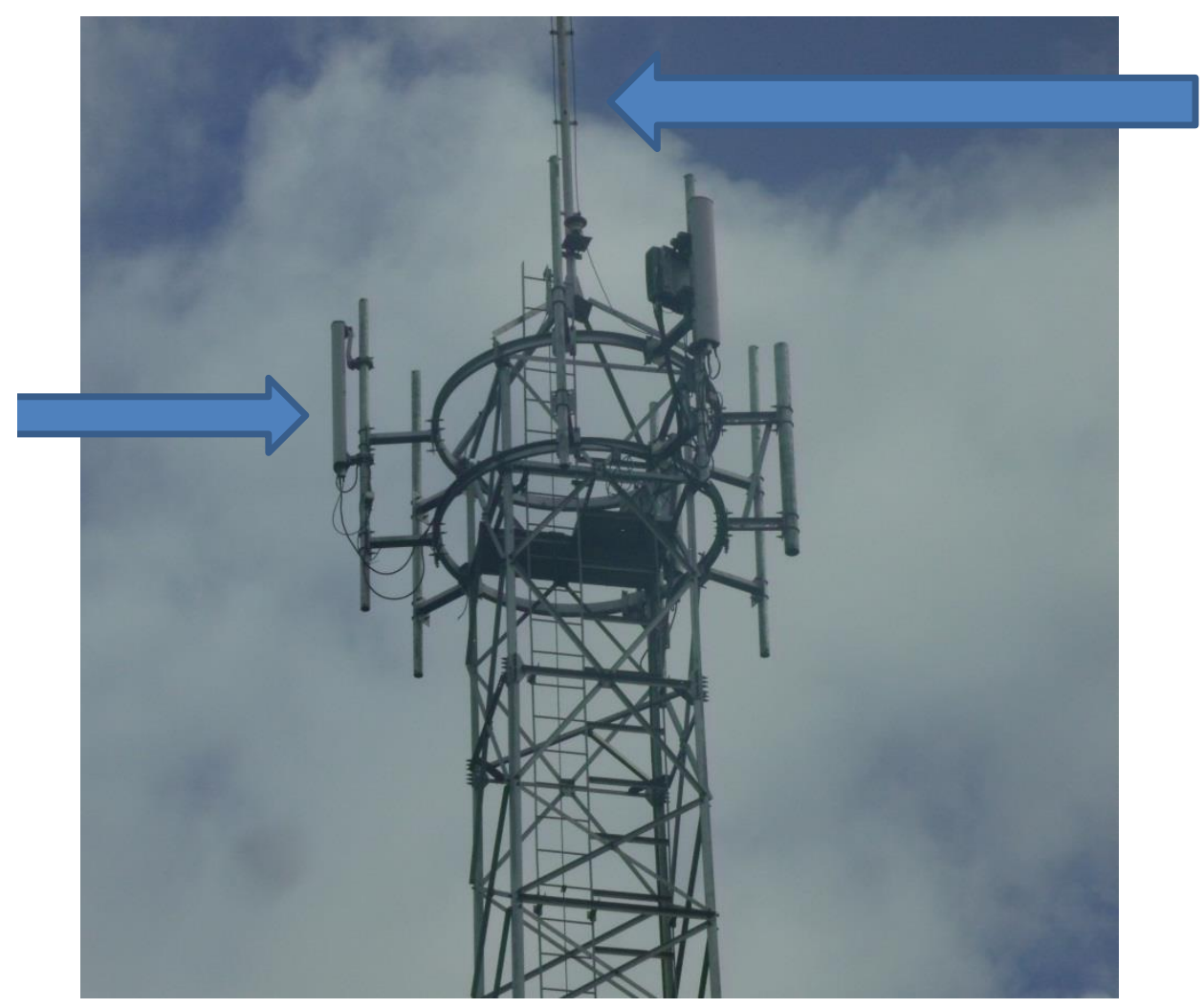

PARA-RAIO

VISADA DA ESTRUTURA

DA TORRE DA ERB

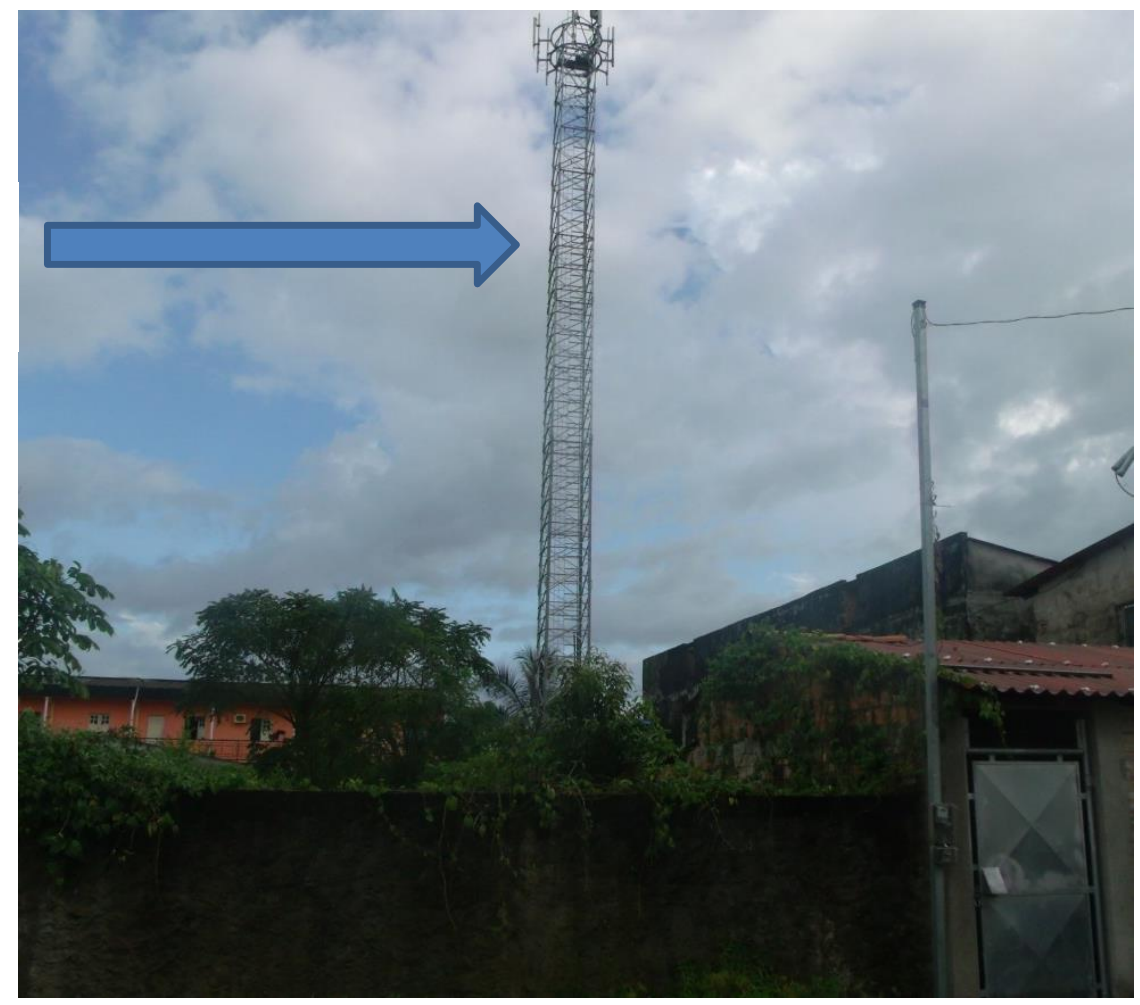




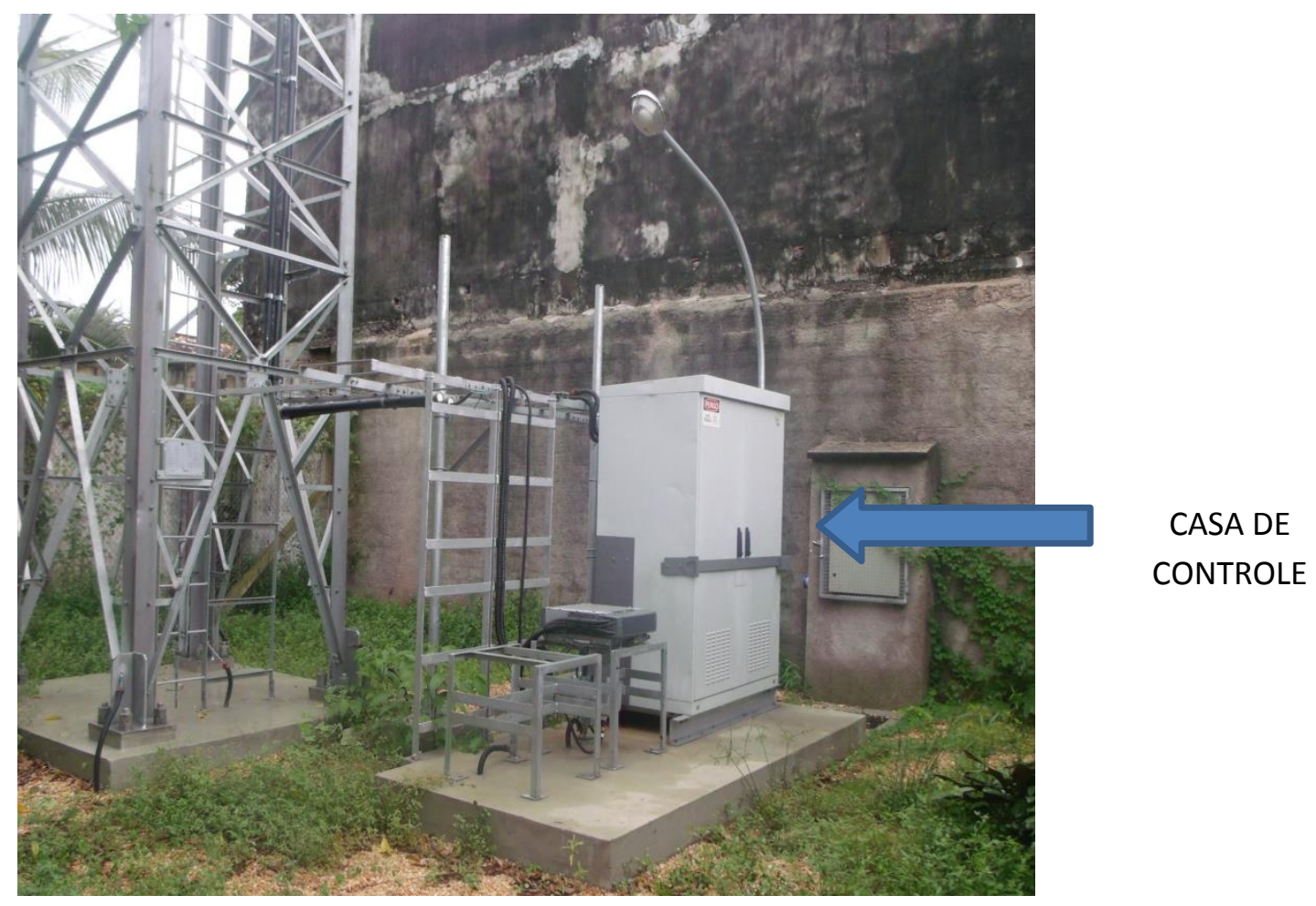


APÊNDICE II: Medições realizadas em escolas

\begin{tabular}{|c|c|c|c|c|}
\hline Bairro & Escola & $\begin{array}{c}\text { Medida do } \\
\text { campo elétrico } \\
(\mathrm{V} / \mathrm{m})\end{array}$ & $\begin{array}{l}\text { Densidade de } \\
\text { potência }(\mathrm{A} / \mathrm{m})\end{array}$ & $\begin{array}{c}\text { Campo magnético } \\
\left(\mathrm{W} / \mathrm{m}^{2}\right) .\end{array}$ \\
\hline Batista campos & TIRADENTES I & 2,264754 & 0,013605068 & 0,006007305 \\
\hline Batista campos & AMAZONAS DE FIGUEIREDO & 1,634052 & 0,007082562 & 0,004334355 \\
\hline Batista campos & ERICO VERISSIMO & 0,351074 & 0,000326931 & 0,000931231 \\
\hline Batista campos & JOSE ALVARES DE AZEVEDO & 1,206711 & 0,003862471 & 0,003200825 \\
\hline Batista campos & TIRADENTES II & 1,057506 & 0,002966363 & 0,002805056 \\
\hline Marambaia & ALMIRANTE TAMANDARÉ & 0,97093 & 0,002500544 & 0,002575411 \\
\hline Marambaia & HILDA VIEIRA & 0,333038 & 0,000294202 & 0,00088339 \\
\hline Marambaia & LEONOR NOGUEIRA & 0,311004 & 0,000256561 & 0,000824944 \\
\hline Marambaia & $\begin{array}{l}\text { FRANCISCO DA SILVA } \\
\text { NUNES }\end{array}$ & 0,380054 & 0,000383133 & 0,001008101 \\
\hline Marambaia & CORNELIO DE BARROS & 0,231657 & 0,000142347 & 0,000614475 \\
\hline Marambaia & VIRGINIA ALVES DE CUNHA & 0,41613 & 0,000459321 & 0,001103793 \\
\hline Marambaia & TEMISTICLES DE ARAUJO & 0,140069 & $5,20406 \mathrm{E}-05$ & 0,000371536 \\
\hline Marambaia & CARLOS GUIMARAES & 0,428836 & 0,000487799 & 0,001137496 \\
\hline Marambaia & DUQUE DE CAXIAS & 0,026049 & 1,79987E-06 & $6,90955 \mathrm{E}-05$ \\
\hline Sacramenta & ROSALINA & 0,476477 & 0,000602202 & 0,001263865 \\
\hline Sacramenta & GRAZIELA & 0,282021 & 0,00021097 & 0,000748066 \\
\hline Sacramenta & SANTA LUZIA & 0,314775 & 0,00026282 & 0,000834947 \\
\hline Sacramenta & EMILIANA SARMENTO & 0,979376 & 0,002544237 & 0,002597814 \\
\hline Sacramenta & ACY DE JESUS & 0,456702 & 0,000553254 & 0,001211411 \\
\hline Cremação & STELIO MARAJO & 0,332419753 & 0,000293111 & 0,00088175 \\
\hline Cremação & RUTH ROSITA & 1,268544153 & 0,004268446 & 0,003364839 \\
\hline Cremação & JOÃO RENATO FRANCO & 1,174258373 & 0,003657514 & 0,003114744 \\
\hline Cremação & MARIO CHERMONT & 0,47793578 & 0,000605896 & 0,001267734 \\
\hline Cremação & AMILCAR TOPASSU & 0,995701275 & 0,002629764 & 0,002641117 \\
\hline Cremação & NORMA MORHY & 0,418032787 & 0,000463532 & 0,00110884 \\
\hline Nazaré & ULISSES GUIMARAES & 0,396085343 & 0,000416137 & 0,001050624 \\
\hline Nazaré & ORLANDO BITTAR & 0,807728238 & 0,00173057 & 0,002142515 \\
\hline Nazaré & DEODORO DE MENDONÇA & 0,496567944 & 0,000654058 & 0,001317156 \\
\hline Nazaré & PINTO MARQUES & 1,554201878 & 0,006407277 & 0,004122551 \\
\hline Nazaré & COLÉGIO NAZARÉ & 1,227383721 & 0,003995944 & 0,00325566 \\
\hline Nazaré & GENTIL BITENCOURT & 0,963404826 & 0,002461933 & 0,00255545 \\
\hline Pedreira & CESEP E MARAJOARA & 0,623603808 & 0,001031516 & 0,001654122 \\
\hline Pedreira & JUSTO ALDEIA SALESIANO & 1,007513594 & 0,00269253 & 0,00267245 \\
\hline Pedreira & $\begin{array}{l}\text { APERECIDA NILTON } \\
\text { DONATILA }\end{array}$ & 0,620869978 & 0,001022492 & 0,00164687 \\
\hline Pedreira & $\begin{array}{l}\text { SOSSEGO PINAGES VERA } \\
\text { MAGALHAES }\end{array}$ & 0,79781394 & 0,001688348 & 0,002116217 \\
\hline Reduto & BENJAMIN CONSTANT & 0,262119914 & 0,000182246 & 0,000695278 \\
\hline Reduto & CEMPE E PETELECO & 0,740473538 & 0,001454379 & 0,001964121 \\
\hline Cidade velha & CARMO & 0,617854 & 0,001012582 & 0,00163887 \\
\hline Cidade velha & GENERAL GURJÃO & 0,587559 & 0,000915718 & 0,001558512 \\
\hline Cidade velha & CASTELO BRANCO & 0,998413 & 0,002644107 & 0,00264831 \\
\hline Cidade velha & RUI BARBOSA & 0,341247 & 0,000308885 & 0,000905164 \\
\hline Guamá & JOSE BONIFACIO & 0,772751 & 0,001583937 & 0,002049737 \\
\hline
\end{tabular}




\begin{tabular}{|c|c|c|c|c|}
\hline Guamá & PAULO MARANHAO & 0,108679 & 3,13292E-05 & 0,000288273 \\
\hline Guamá & BENTO VX & 0,830716 & 0,001830475 & 0,002203491 \\
\hline Guamá & NS GRAÇAS & 0,689409 & 0,001260702 & 0,001828671 \\
\hline Guamá & UNIVERSO MUNDURUCUS & 0,417946 & 0,000463339 & 0,00110861 \\
\hline Guamá & SANTOS DUMOND & 0,596689 & 0,000944397 & 0,001582729 \\
\hline Guamá & XV DE OUTUBRO & 0,755035 & 0,001512143 & 0,002002745 \\
\hline Guamá & ALEXANDRE ZACARIAS & 1,55005 & 0,00637309 & 0,004111538 \\
\hline Guamá & BARAO DE IGARAPE MIRIM & 0,7276 & 0,001404249 & 0,001929973 \\
\hline Guamá & CELINA ANGLADA & 0,526835 & 0,00073622 & 0,00139744 \\
\hline Guamá & $\begin{array}{l}\text { FREI DANIEL E PADRE } \\
\text { LEANDRO PINHEIRO }\end{array}$ & 0,642995 & 0,001096665 & 0,001705557 \\
\hline Guamá & ROSA GATORNO & 0,255305 & 0,000172893 & 0,000677202 \\
\hline Guamá & BIRA BARBOSA & 0,049424 & $6,47939 \mathrm{E}-06$ & 0,000131098 \\
\hline Guamá & HUMBERTO DE CAMPOS & 0,364005 & 0,000351458 & 0,000965531 \\
\hline Guamá & MADRE ZARIFE & 0,497646 & 0,000656901 & 0,001320016 \\
\hline Marco & JARBAS E PEDRO II & 0,953762 & 0,002412896 & 0,002529873 \\
\hline Marco & ACATAUAÇU & 0,755817 & 0,001515277 & 0,00200482 \\
\hline Marco & YOLANDA & 0,434052 & 0,000499738 & 0,001151332 \\
\hline Marco & LAURO SODRE & 0,910739 & 0,002200121 & 0,002415753 \\
\hline Marco & ALBANISIA DE OLIVEIRA & 0,165389 & $7,25558 \mathrm{E}-05$ & 0,000438698 \\
\hline Marco & IFPA & 1,265262 & 0,004246387 & 0,003356133 \\
\hline marco & SABER & 1,789643 & 0,008495549 & 0,004747064 \\
\hline marco & RUHT PASSARINHO & 1,335347 & 0,004729845 & 0,003542034 \\
\hline Marco & SOUSA FRANCO & 0,647935 & 0,00111358 & 0,00171866 \\
\hline Jurunas & ARTHUR PORTO & 0,67 & 0,001190716 & 0,001777188 \\
\hline Jurunas & CAMILO SALGADO & 1,92 & 0,009778249 & 0,005092838 \\
\hline Jurunas & MARIA LUIZA VELLA ALVES & 0,7 & 0,001299735 & 0,001856764 \\
\hline Jurunas & MORADORES VILLA NOVA & 0,97 & 0,002495756 & 0,002572944 \\
\hline Jurunas & PADRE BENEDITO CHAVES & 2,08 & 0,011475862 & 0,005517241 \\
\hline Jurunas & PLACIDA CARDOSO & 0,61 & 0,000987003 & 0,001618037 \\
\hline Jurunas & SÃO PIO X & 0,83 & 0,001827321 & 0,002201592 \\
\hline Jurunas & $\begin{array}{l}\text { VEREADOR GONCALO } \\
\text { DUARTE }\end{array}$ & 0,29 & 0,000223077 & 0,000769231 \\
\hline COMÉRCIO & $\begin{array}{l}\text { DAVID SALOMÃO } \\
\text { MUFARREJ }\end{array}$ & 1,01549774 & 0,002735373 & 0,002693628 \\
\hline COMÉRCIO & IEP & 0,56202632 & 0,000837861 & 0,001490786 \\
\hline COMÉRCIO & CIEES & 0,15502577 & $6,3748 \mathrm{E}-05$ & 0,000411209 \\
\hline CONDOR & ANTONIA PAES DA SILVA & 1,24411429 & 0,004105624 & 0,003300038 \\
\hline CONDOR & MONSENHOR AZEVEDO & 0,52709079 & 0,000736936 & 0,001398119 \\
\hline CONDOR & EDGARD PINHEIRO PORTO & 1,46809942 & 0,005717018 & 0,003894163 \\
\hline TERRA FIRME & BRIGADEIRO FONTENELE & 0,43275229 & 0,000496749 & 0,001147884 \\
\hline TERRA FIRME & $\begin{array}{l}\text { CELSO MALCHER } \\
\text { ELETRONORTE }\end{array}$ & 0,10202247 & $2,7609 \mathrm{E}-05$ & 0,000270617 \\
\hline TERRA FIRME & FONTE VIVA & 0,40337349 & 0,000431592 & 0,001069956 \\
\hline TERRA FIRME & MARIO BARBOSA & 0,92473418 & 0,002268258 & 0,002452876 \\
\hline TERRA FIRME & MATEUS DO CARMO & 0,04200946 & $4,68115 \mathrm{E}-06$ & 0,000111431 \\
\hline TERRA FIRME & NPI & 0,26377528 & 0,000184555 & 0,000699669 \\
\hline TERRA FIRME & UFRA & 0,00010846 & $3,12031 \mathrm{E}-11$ & $2,87692 \mathrm{E}-07$ \\
\hline TERRA FIRME & VIRGÍLIO LIBONATI & 0,04271226 & 4,83909E-06 & 0,000113295 \\
\hline UMARIZAL & DR. FREITAS E APAE & 0,2957425 & 0,000231999 & 0,000784463 \\
\hline
\end{tabular}




\begin{tabular}{|l|l|r|r|r|} 
UMARIZAL & FELIPE SMALDONE & 0,47872017 & 0,000607886 & 0,001269815 \\
\hline UMARIZAL & $\begin{array}{l}\text { PAULA FRANCINETE E SÃO } \\
\text { VICENTE }\end{array}$ & 0,24925926 & 0,000164802 & 0,000661165 \\
\hline UMARIZAL & WALDEMAR RIBEIRO E & & & \\
\hline TELÉGRAFO & SAÚDE DA CRIANÇA & 1,52744681 & 0,006188578 & 0,004051583 \\
\hline TELÉGRAFO & JUGUSTO MONTENEGRO & 0,46478166 & 0,000573003 & 0,001232843 \\
\hline TELÉGRAFO & SANTO AFONSO & 0,35578261 & 0,000335759 & 0,00094372 \\
\hline SOUZA & AUSTERIO E ANESIA & 0,79296296 & 0,001667879 & 0,00210335 \\
\hline SOUZA & CORDEIRO DE FARIAS & 0,71990172 & 0,001374691 & 0,001909554 \\
\hline SOUZA & COSTA E SILVA & 0,43626536 & 0,000416515 & 0,001051102 \\
\hline SOUZA & FUNDAÇÃO PESTALOZZI & 0,42329238 & 0,00050922 & 0,001162203 \\
\hline SOUZA & JARBAS PASSARINHO & 0,00362637 & $3,48821 \mathrm{E}-08$ & 0,001122791 \\
\hline SOUZA & PEDRO PEDROSO & 0,004544545 & $5,47822 \mathrm{E}-08$ & $1,20545 \mathrm{E}-065$ \\
\hline & PREVENTÓRIO SANTA & & & \\
\hline SOUZA & TEREZINHA & 0,15407862 & $6,29714 \mathrm{E}-05$ & 0,000408697 \\
\hline SOUZA & REGO BARROS & 0,78721675 & 0,001643794 & 0,002088108 \\
\hline SÃO BRAZ & AUGUSTO MEIRA & 1,242948 & 0,00409793 & 0,003296944 \\
\hline SÃO BRAZ & ANIBAL MENDONÇA & 0,54293 & 0,000781891 & 0,001440133 \\
\hline SÃO BRAZ & LUIZ OTÁVIO PEREIRA & 0,54293 & 0,000781891 & 0,001440133 \\
\hline SÃO BRAZ & AUGUSTO OLIMPO & 0,764477 & 0,001550199 & 0,00202779 \\
\hline SÃO BRAZ & UNIVERSO 9 DE JANEIRO & 0,766008 & 0,001556414 & 0,002031851 \\
\hline
\end{tabular}


Apêndice III: Medições realizadas em hospitais

\begin{tabular}{|l|l|r|r|r|}
\hline Bairro & Hospital & $\begin{array}{l}\text { Medida do campo } \\
\text { elétrico }(\mathrm{V} / \mathrm{m})\end{array}$ & $\begin{array}{l}\text { Densidade de } \\
\text { potência }(\mathrm{A} / \mathrm{m})\end{array}$ & $\begin{array}{l}\text { Campo magnético } \\
\left(\mathrm{W} / \mathrm{m}^{2}\right) .\end{array}$ \\
\hline Terra Firme & $\begin{array}{l}\text { Hospital Betina } \\
\text { Ferro de Souza }\end{array}$ & 0,524352022 & 0,000729297 & 0,001390854 \\
\hline UMARIZAL & PSM 14 & 0,503423273 & 0,000672241 & 0,00133534 \\
\hline & $\begin{array}{l}\text { Maternidade } \\
\text { Saúde da }\end{array}$ & 1,532473573 & 0,006229377 & 0,004064917 \\
\hline UMARIZAL & Criança & 0,514794326 & 0,000702953 & 0,001365502 \\
\hline UMARIZAL & Santa Casa & 0,734604317 & 0,001431415 & 0,001948553 \\
\hline MARCO & $\begin{array}{l}\text { Saude da } \\
\text { Mulher }\end{array}$ & & & \\
\hline
\end{tabular}


APÊNDICE IV: Mapa de distribuição das ERBS em Belém

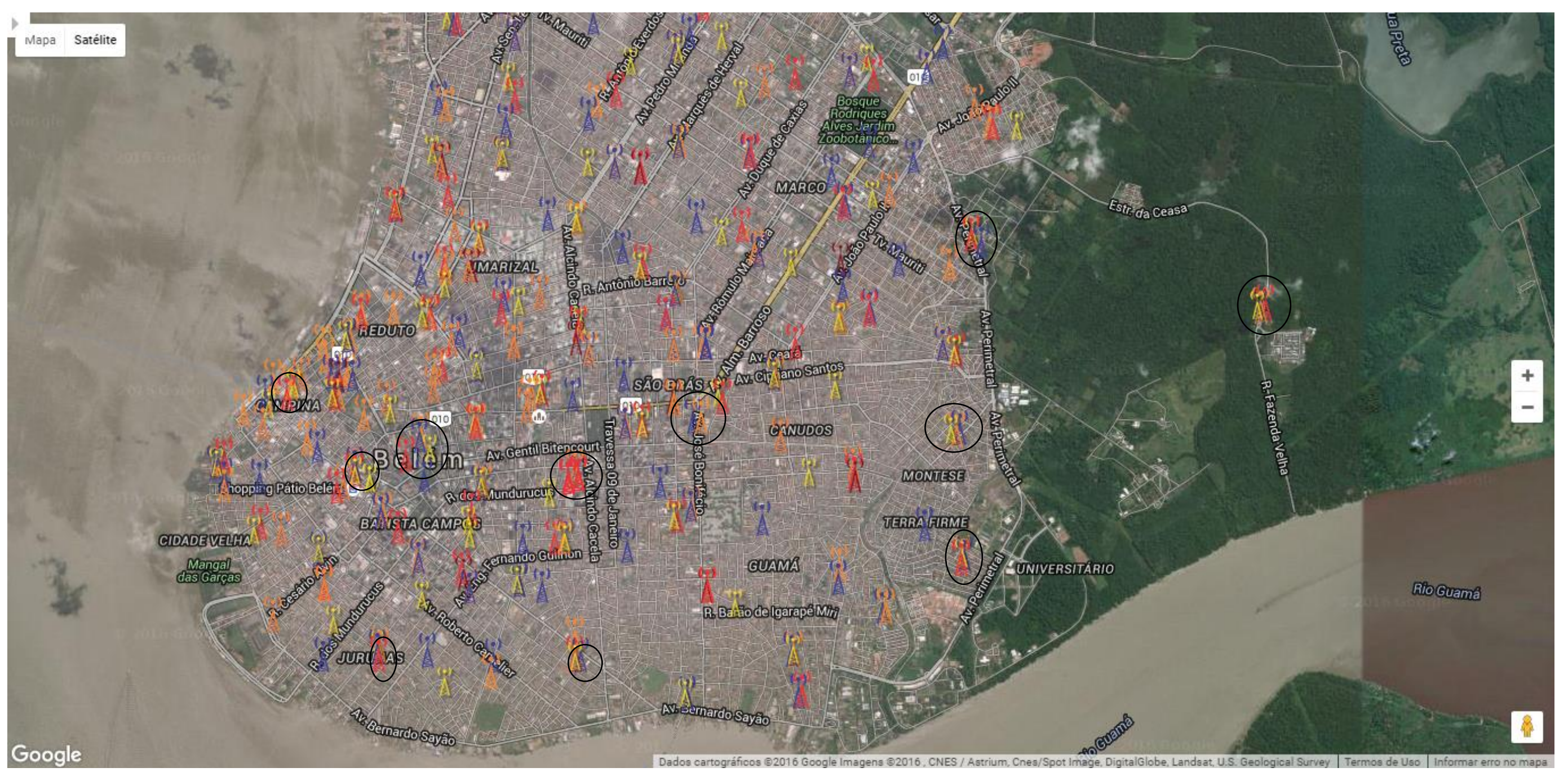

